\title{
DETC2001/VIB-21390
}

\section{ACTIVE CONTROL OF ROTOR VIBRATIONS BY MEANS OF PIEZOELECTRIC ACTUATORS}

\author{
Costantino Carmignani/University of Pisa, Italy
}

Paola Forte/University of Pisa, Italy

Emiliano Rustighi/University of Pisa, Italy

\begin{abstract}
This work deals with the development of an adaptive hydrodynamic bearing made of a mobile housing mounted on piezoelectric actuators. The device is placed near one of the two bearings supporting a slender shaft. Imposing a harmonic displacement on the mobile bearing, in two orthogonal directions, a rotating force, and hence a correcting moment, can be produced on the shaft so as to reduce the bending caused by the unbalance. The first tests carried out are encouraging and the agreement of experimental and numerical results is satisfactory.
\end{abstract}

\section{INTRODUCTION}

The unbalance forces are the main source of vibrations in rotating machines, but a perfect balance is almost impossible to reach, and in any case very expensive. Moreover the distribution of unbalance can change in time because of wear or depositions all machines are subjected to. The most usual way to contrast the losses of balance is to increase damping in order to absorb a greater amount of dynamical energy. Nevertheless in many cases, to obtain specific performances of the rotors in a wider range of speeds, the use of passive elements (such as absorbers and dampers) and/or the optimization of the design parameters are not sufficient, and active elements are needed. The main advantage of active control, versus passive, is the versatility in adapting to several load conditions and configurations of the machine. Moreover the control system operates only if necessary, modifying only some parameters of the controlled system. On the contrary the use of a passive damper involves a continuous dissipation of energy in all the working conditions of the machine with the consequence of a decreasing efficiency.

At present, in order to realize an active control system, magnetic bearings are the mostly used devices but their use in the industrial field is still limited for their low stiffness and for the need of additional conventional bearings for fault emergency. Many papers, available in the technical literature, describe other active control devices that use different types of actuators such as pneumatic, hydraulic or electric-hydraulic ones [1-4], or induced-currents generators of force, but no one of these systems has shown a remarkable superiority above the others.

The use of piezoelectric actuators as active vibrations dampers in rotating machines has been considered in the past [5,6]. In particular, some experiences have been carried out on rotors mounted on two rolling bearings, each one supported, with rubber pads in between, by four piezoelectric actuators fixed to the frame. In spite of their promising results, their application to the control of rotor dynamics has not had any following but for few mentions in some theoretical works $[7,8]$ where they are assumed to be applied in active balancing of rigid rotors. The wider variety of presently available commercial piezoelectric translators has certainly contributed to a renewed interest for their use in vibration control.

The objective of this work was fundamentally the realization of a test rig to verify the feasibility of active control of vibrations in rotor dynamics by an alternative technique to magnetic bearings. In particular the possibility of reducing the amplitude of vibrations of a flexible statically unbalanced rotor within acceptable levels was investigated. That was carried out by designing a hydrodynamic bearing made active through a 
high frequency control. The active bearing is made of a mobile housing moved by two piezoelectric actuators. The actuators, arranged at $90^{\circ}$ on a plane perpendicular to the shaft axis, exert two sinusoidal forces with a tuned phase angle so as to produce a balancing or, in alternative, a dampening effect. The active bearing is placed nearby one of the two conventional ones supporting the shaft, so that it can generate a straightening moment at one end of the rotor. The resulting increase of force transmitted to the foundation is tolerated for a decrease of the rotor displacement. The device has been interfaced to Bently Nevada Rotor Kit test bench (Fig. 1), that in spite of its reduced dimensions $(775 \times 150 \times 340 \mathrm{~mm})$, makes the simulation of the behavior of large rotating machines possible.

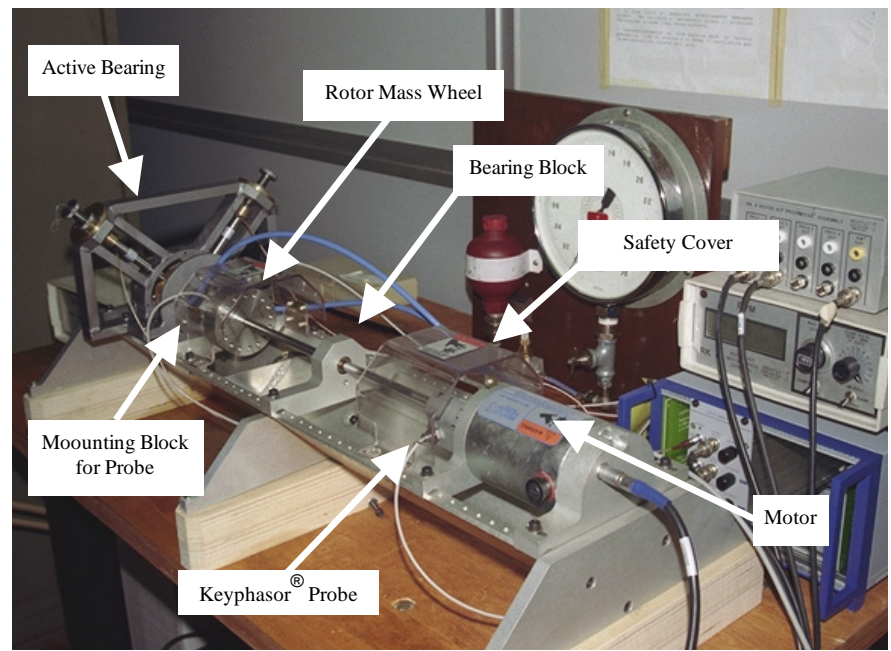

Fig. 1. Modified Bently Nevada Rotor Kit Bench

The control technique to be adopted with this kind of device is a periodic feedforward one, with compensation of the disturbance. It has been taken into consideration because it has the advantage of not involving modifications in the characteristics of the controlled loop and therefore doesn't produce problems of instability usually present in feedback control $[9,10]$.

\section{NOMENCLATURE}

$c$

$c_{x x}, c_{x y}, c_{y x}, c_{y y}$

$F_{c x}, F_{c y}$

$k_{11}, k_{12}, k_{21}, k_{22}$

$k_{x x}, k_{x y}, k_{y x}, k_{y y}$

$m$

$x_{1}, y_{1}$

$x_{2}, y_{2}$

$x_{3}, y_{3}$ structural and aerodynamic damping

hydrodynamic bearing linearized coefficients

components of centrifugal force

elastic constants of the shaft

hydrodynamic bearing linearized elastic constants

lumped mass

coordinates of lumped mass

active bearing journal coordinates

active bearing housing coordinates
ANALYTICAL STUDY AND NUMERICAL SIMULATION

The analysis of feasibility of the control system was made by means of a numerical simulation. Referring to the structure of the Rotor Kit bench, a model of the system was defined, made of a slender shaft carrying a lumped mass on its midspan, supported at the ends by two fixed bearings. The rotor was also supported, as shown in Fig. 2, by a moving hydrodynamic bearing: the active bearing.

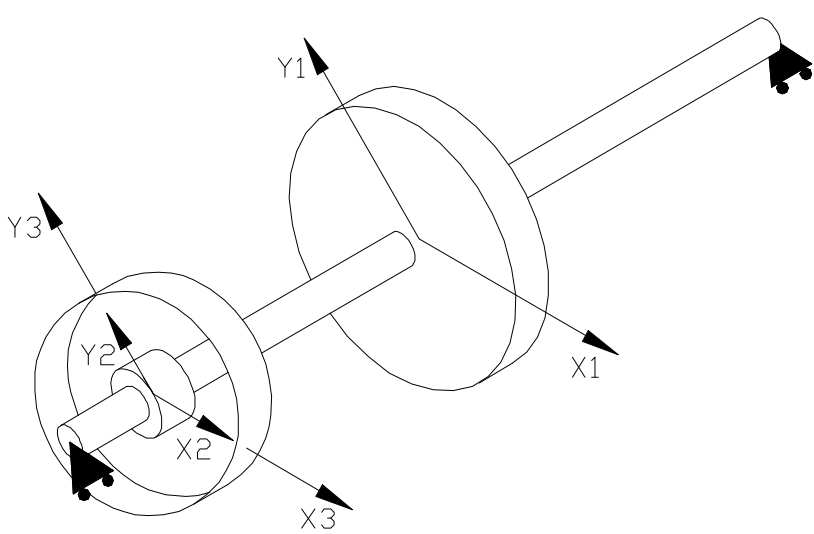

Fig. 2. Model of rotor with moving bearing system

The structural and aerodynamic damping $c$ was introduced to take into account the real damping conditions and to avoid infinite transients. The equations of motion of the shaft, and the equations of balance of the elastic reactions of the shaft and those of the bearing [11] are:

$$
\begin{gathered}
m \ddot{x}_{1}+c \dot{x}_{1}+k_{11} x_{1}+k_{12} x_{2}=F_{c x} \\
m \ddot{y}_{1}+c \dot{y}_{1}+k_{11} y_{1}+k_{12} y_{2}=F_{c y} \\
{\left[\begin{array}{cc}
c_{x x} & c_{x y} \\
c_{y x} & c_{y y}
\end{array}\right]\left\{\begin{array}{l}
\dot{x}_{2}-\dot{x}_{3} \\
\dot{y}_{2}-\dot{y}_{3}
\end{array}\right\}+\left[\begin{array}{ll}
k_{x x} & k_{x y} \\
k_{y x} & k_{y y}
\end{array}\right]\left\{\begin{array}{l}
x_{2}-x_{3} \\
y_{2}-y_{3}
\end{array}\right\}=} \\
=-\left[\begin{array}{cc}
k_{21} & 0 \\
0 & k_{21}
\end{array}\right]\left\{\begin{array}{l}
x_{1} \\
y_{1}
\end{array}\right\}-\left[\begin{array}{cc}
k_{22} & 0 \\
0 & k_{22}
\end{array}\right]\left\{\begin{array}{l}
x_{2} \\
y_{2}
\end{array}\right\}
\end{gathered}
$$

The linearized bearing coefficients were obtained using the equation of Ocvirk's short bearing [12] for an eccentricity ratio of 0.5 . In order to avoid instability the active bearing should not operate at low eccentricity ratios. The use of a tilting pad bearing could remove this limitation.

The elastic constants of the shaft which is in a statically indeterminate configuration were determined by means of a finite element method program. The static indeterminacy is actually necessary in order to generate the straightening moment at one end of the shaft.

Hence the matrices of the state equation (2) were obtained in order to perform the analysis of controllability and observability in Matlab $^{\circledR}$ (the system is observable and controllable). Moreover a stability analysis was performed as 
well (the system is stable in the range of rotating speeds of the Rotor Kit).

$$
\begin{aligned}
& {\left[\begin{array}{cccccc}
0 & 0 & 1 & 0 & 0 & 0 \\
0 & 0 & 0 & 1 & 0 & 0 \\
-\frac{k_{11}}{m} & 0 & -\frac{c}{m} & 0 & -\frac{k_{12}}{m} & 0 \\
0 & -\frac{k_{11}}{m} & 0 & -\frac{c}{m} & 0 & -\frac{k_{12}}{m} \\
a_{11} & a_{12} & 0 & 0 & b_{11} & b_{12} \\
a_{21} & a_{22} & 0 & 0 & b_{21} & b_{22}
\end{array}\right]\left\{\begin{array}{l}
x_{1} \\
y_{1} \\
\dot{x}_{1} \\
\dot{y}_{1} \\
x_{2} \\
y_{2}
\end{array}\right\}+} \\
&+\left[\begin{array}{cccccc}
0 & 0 & 0 & 0 & 0 & 0 \\
0 & 0 & 0 & 0 & 0 & 0 \\
\frac{1}{m} & 0 & 0 & 0 & 0 & 0 \\
0 & 1 & 0 & 0 & 0 & 0 \\
0 & 0 & d_{11} & d_{12} & 1 & 0 \\
0 & 0 & d_{21} & d_{22} & 0 & 1
\end{array}\right]\left\{\begin{array}{l}
F_{c x} \\
F_{c y} \\
x_{3} \\
y_{3} \\
\dot{x}_{3} \\
\dot{y}_{3}
\end{array}\right\} \\
&\left\{\begin{array}{l}
x_{1} \\
y_{1} \\
x_{2} \\
y_{2}
\end{array}\right\}= {\left[\begin{array}{llllll}
1 & 0 & 0 & 0 & 0 & 0 \\
0 & 1 & 0 & 0 & 0 & 0 \\
0 & 0 & 0 & 0 & 1 & 0 \\
0 & 0 & 0 & 0 & 0 & 1
\end{array}\right]\left\{\begin{array}{l}
x_{1} \\
y_{1} \\
\dot{x}_{1} \\
\dot{y}_{1} \\
x_{2} \\
y_{2}
\end{array}\right\} }
\end{aligned}
$$

with:

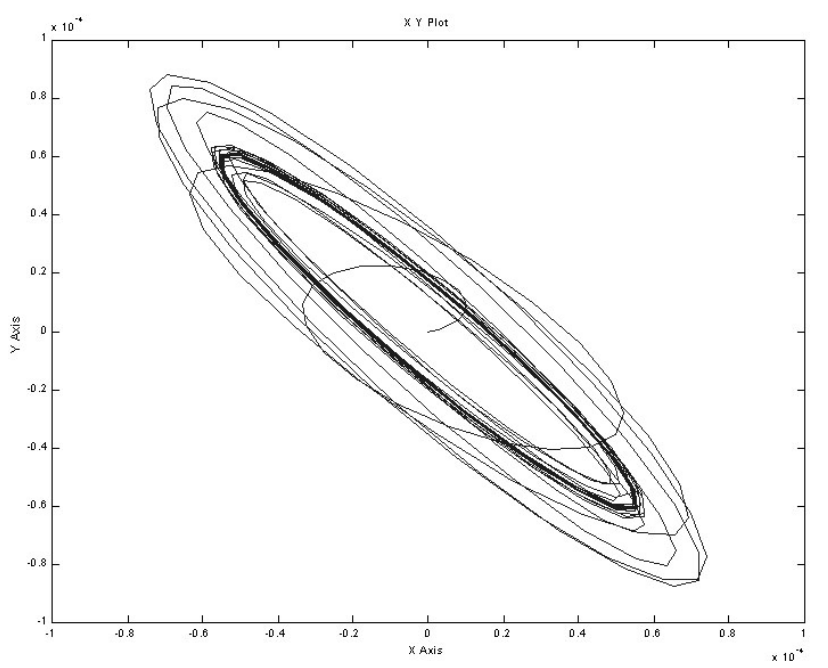

Fig. 3. Orbits of lumped mass and journal at $5000 \mathrm{rpm}$, fixed housing
The first simulation was carried out fixing the housing of the active bearing and rotating the shaft at $5000 \mathrm{rpm}$ : in this case the lumped mass moves in an orbit with a maximum radius of $60 \mu \mathrm{m}$ while the journal moves in an orbit with a maximum radius of $20 \mu \mathrm{m}$ (Fig. 3). Then the behavior of this system was simulated by imposing to the active bearing housing different circular trajectories with various phase angles between them and the centrifugal force. In particular, an interesting case is that in which the active bearing housing moves in a circular trajectory with a radius of $25 \mu \mathrm{m}$ synchronous with the centrifugal force and with a phase angle of $180^{\circ}$. In this case, the lumped mass has a flat elliptical orbit the greater semi-axis of which is $80 \mu \mathrm{m}$. At the same time, the journal moves in a flat elliptical orbit the greater semi-axis of which is about $28 \mu \mathrm{m}$ (Fig. 4).

Since the reduction of the orbit was noticed in only one direction, it seemed reasonable to force the housing to move in an elliptical trajectory with axes of 50 and $10 \mu \mathrm{m}$ : the effects obtained by an appropriate displacement of the active housing are evident (Fig. 5): the lumped mass orbit has a maximum radius of $40 \mu \mathrm{m}$ while the journal orbit has a maximum radius of $10 \mu \mathrm{m}$.

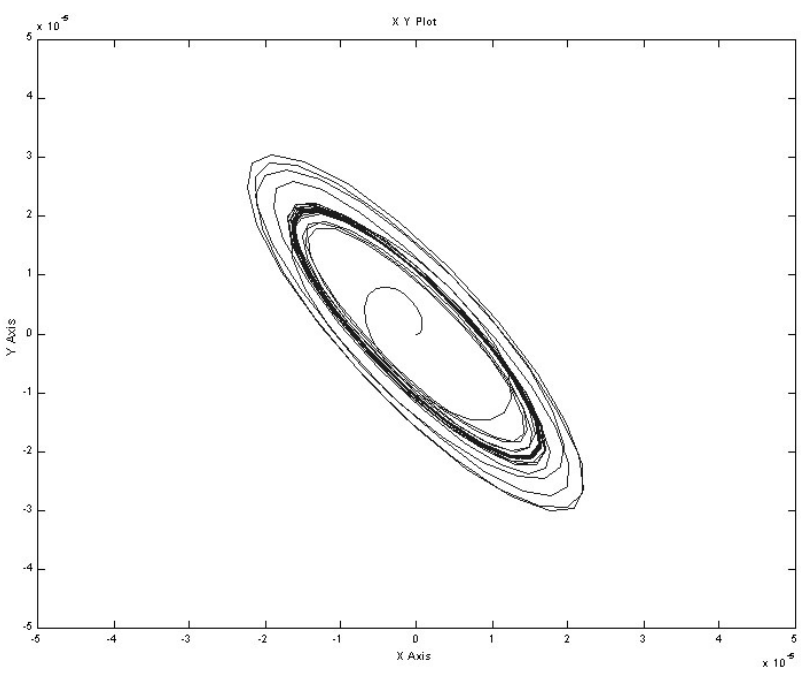



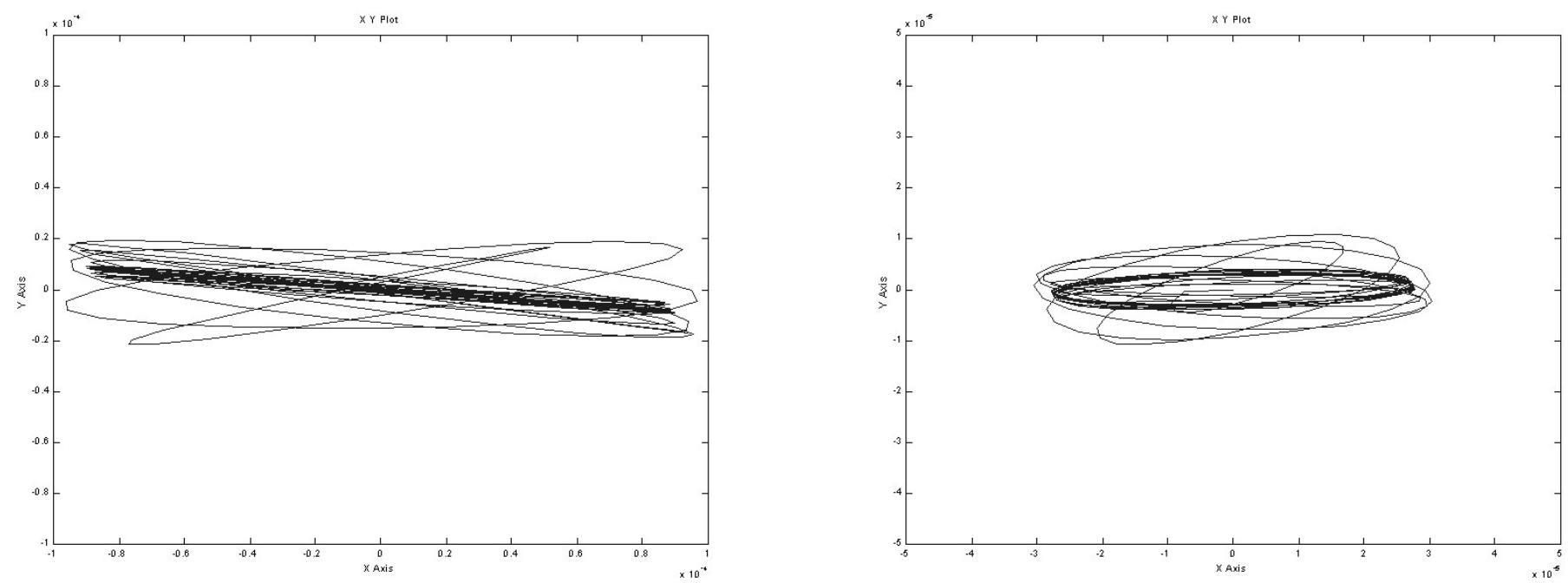

Fig. 4. Orbits of lumped mass and journal at $5000 \mathrm{rpm}$, housing moving in a circular trajectory
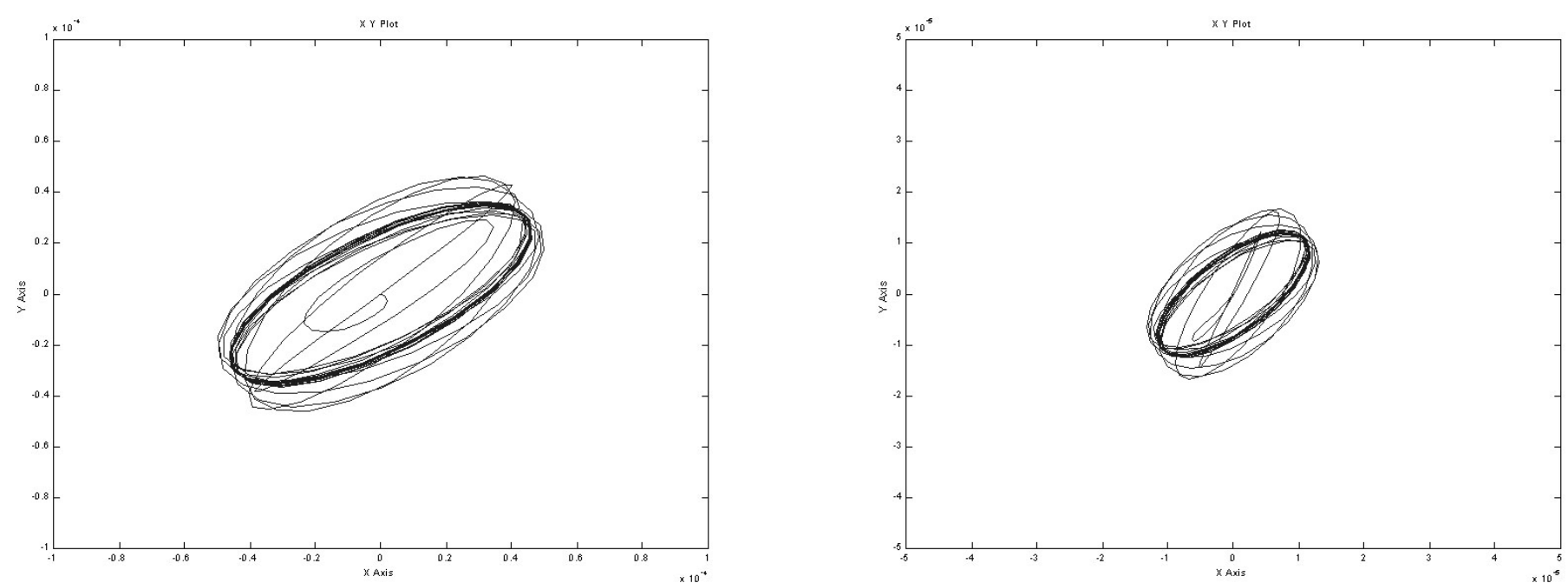

Fig. 5. Orbits of lumped mass and journal at $\mathbf{5 0 0 0} \mathrm{rpm}$, housing moving in an elliptical trajectory

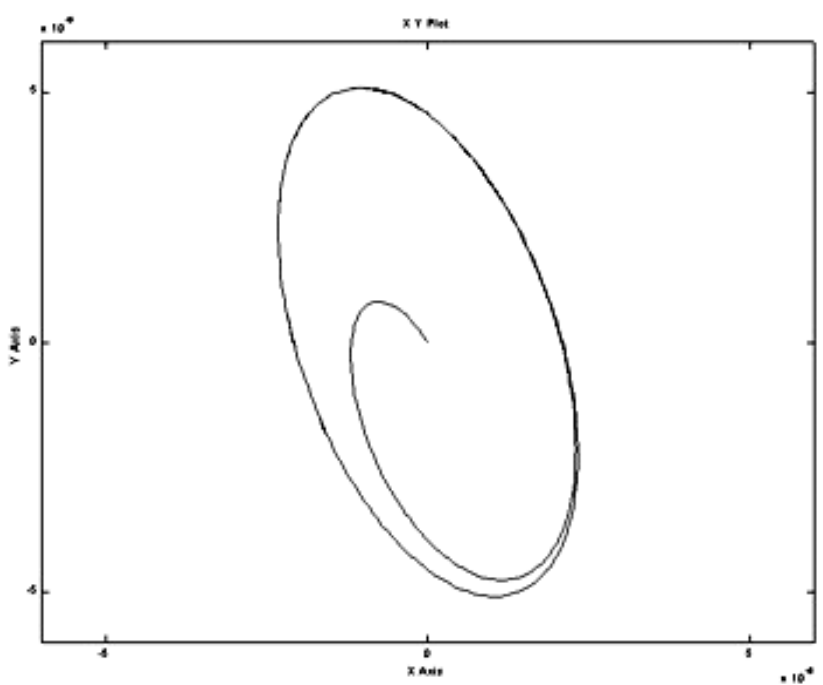

Finally the optimum orbit in which the housing must move in order to have no displacement of lumped mass was calculated numerically. To do that the corresponding displacement values in the state equation (2) were set equal to zero. Carrying out a simulation, the elliptical curve of Fig. 6 was obtained.

\section{DESCRIPTION OF THE TEST RIG}

The rotor, which was supplied with the Rotor Kit bench of Bently Nevada, is a slender $10 \mathrm{~mm}$ diameter shaft, $560 \mathrm{~mm}$ long. One or two discs of $0.8 \mathrm{~kg}$ each can be fixed on the shaft. These discs have threaded holes at a radius of $30 \mathrm{~mm}$ in order to create the desired unbalance inserting appropriate screwweights. The shaft, supported by two adjustable oilite bearings, can reach a maximum rotational speed of $10,000 \mathrm{rpm}$. The Rotor Kit bench is equipped with eddy current probes for

Fig. 6. Optimal orbit of housing, obtained by simulation 
relative displacement measurements (3300 NSVTM Probe Proximitor $^{\circledR}$ ) so that the shaft precessional movement can be detected simultaneously in at least two sections.
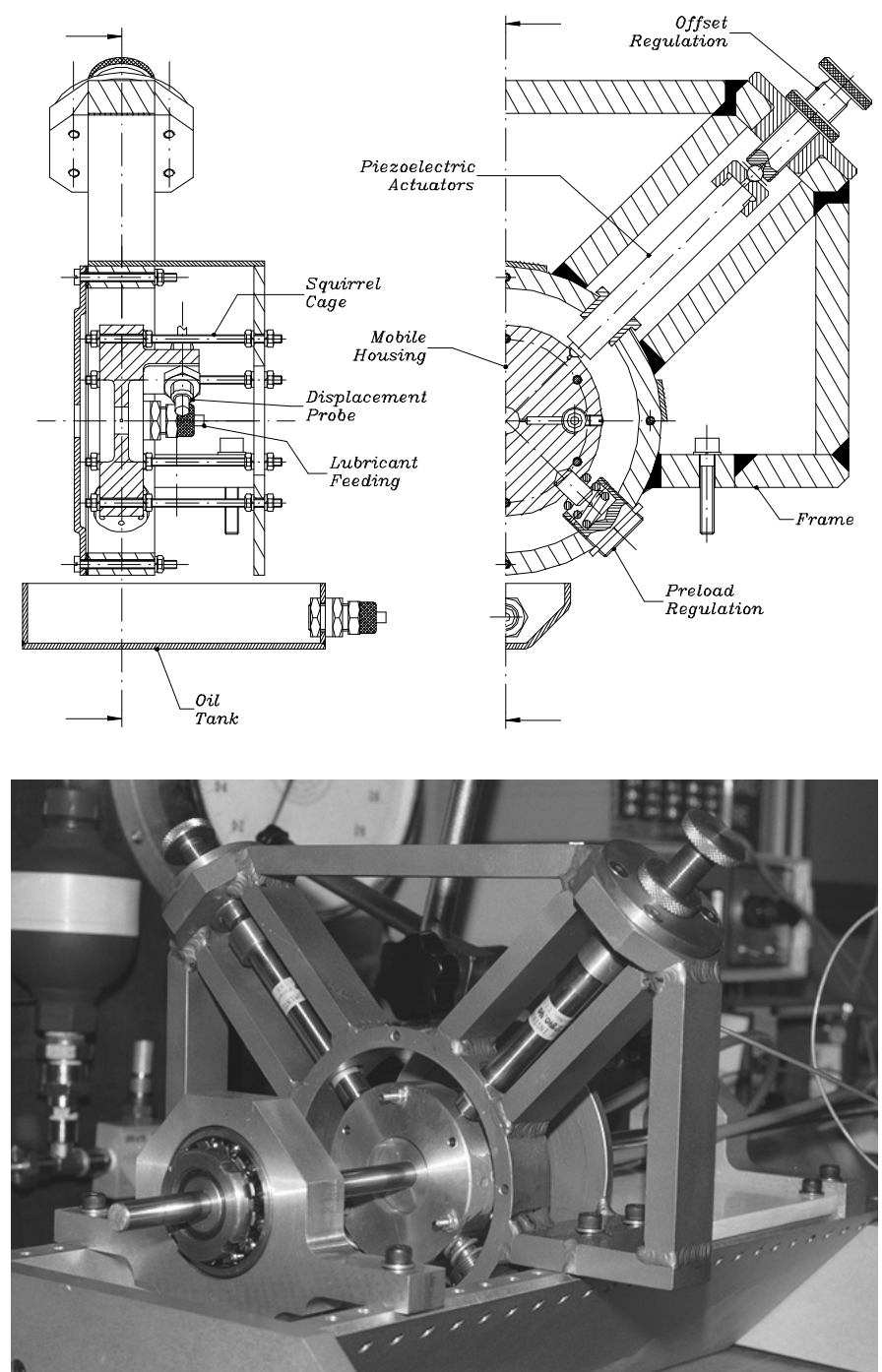

Fig. 7. Active control device: drawing and photo

The active hydrodynamic bearing has a radial clearance of $30 \mu \mathrm{m}$ and a length to diameter ratio of 0.5 . Two piezoelectric actuators (see Tab. 1), set perpendicularly to each other act on the hydrodynamic bearing. This configuration permits to move the housing independently in two perpendicular directions. Therefore it is possible to move the housing along an elliptical trajectory as a result of vector combination of orthogonal harmonic displacements. Since the actuators respond only in expansion, a reaction spring was set against each actuator (Fig. 7). Both spring position and pre-load are adjustable. The hydrodynamic bearing is also constrained against rotation by a squirrel cage which reacts to the oil film friction torque. Lubricant is supplied to the hydrodynamic bearing by two supply holes, placed horizontally, one in front of the other.
The oil pump was part of the equipment of the Rotor Kit bench. Two displacement probes are mounted on the mobile housing in order to measure relative displacements between the shaft and the bearing.

The original Rotor Kit supports presented a too large radial clearance. Consequently the effect of the actuator was nullified by a non negligible shaft displacement inside the oilite bearings. In such condition, it isn't possible to generate a couple of forces so as to bring back the rotating disc to the ideal central position. In order to have no radial displacements of the shaft at its supported end, a support with a reduced radial clearance was realized and placed at the end of the shaft near the mobile hydrodynamic bearing. This support is made of a self-aligning ball bearing, fastened to the shaft trough a conical adapter sleeve. The problem of clearance between the shaft and his supports is critical because of the presence of only one active bearing and of the peculiarity of piezoactuators that develop forces inversely proportional to displacements.

\begin{tabular}{|l|l|}
\hline Open loop travel @ 0 to $100 \mathrm{~V}$ & $\Delta \mathrm{L}_{0}=60 \mu \mathrm{m} \pm 20 \%$ \\
\hline Open loop resolution & $0.6 \mathrm{~nm}$ \\
\hline Stiffness & $\mathrm{k}_{\text {att }}=25 \mathrm{~N} / \mu \mathrm{m} \pm 20 \%$ \\
\hline Maximum force generation & $\mathrm{F}_{\mathrm{MAx}}=1500 \mathrm{~N} \pm 20 \%$ \\
\hline Nominal pull force capacity & $100 \mathrm{~N}$ \\
\hline Nominal push force capacity & $1000 \mathrm{~N}$ \\
\hline Torque limit (at tip) & $350 \mathrm{mNm}$ \\
\hline $\begin{array}{l}\text { Dynamic Operating Current } \\
\text { Coefficient }\end{array}$ & $\mathrm{DOCC}=15 \mu \mathrm{A} /(\mathrm{Hz} \cdot \mu \mathrm{m})$ \\
\hline Unloaded resonant frequency & $\mathrm{f}_{0}=8.5 \mathrm{kHz} \pm 20 \%$ \\
\hline $\begin{array}{l}\text { Standard operating temperature } \\
\text { range }\end{array}$ & -20 to $+80{ }^{\circ} \mathrm{C}$ \\
\hline Weight without cables & $\mathrm{m}_{\text {att }}=54 \mathrm{~g} \pm 5 \%$ \\
\hline Length & $\mathrm{L}=86 \mathrm{~mm} \pm 0.3$ \\
\hline
\end{tabular}

Table 1. Technical data of Physik Instrumente P840.40 piezoelectric actuator

\section{DEFINITION OF EXPERIMENTS}

The control device and the Rotor Kit bench were linked to a personal computer by means of a National Instruments data acquisition board (Fig. 8). Software programs were developed in $L a b V I E W^{\mathrm{TM}}$ environment in order to perform the experiences previously simulated by Simulink ${ }^{\circledR}$ in Matlab ${ }^{\circledR}$. The displacements of the shaft in uncontrolled and controlled operating conditions are acquired by means of VIs (Virtual Instruments). Simultaneously it is possible to acquire the lubricant temperature and to monitor its pressure. All the experimental data are saved in a text file, defined by the user, in a format compatible with any spreadsheet program.

The experimental tests carried out with the active control device followed the simulated ones. The two end supports were 


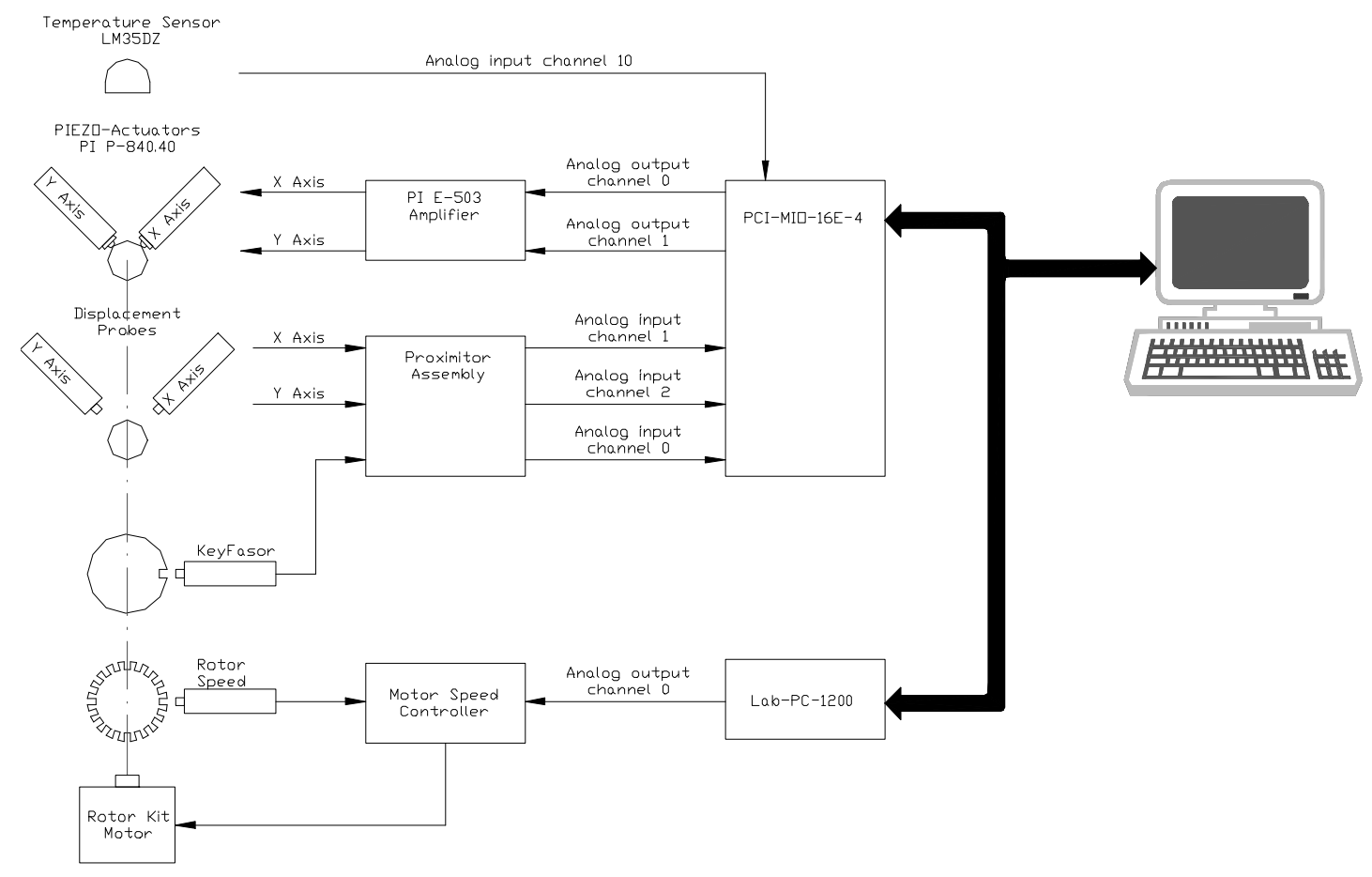

Fig. 8. Schematic of the experimental rig and instrumentation

fixed at $350 \mathrm{~mm}$ from each other and the $0.8 \mathrm{~kg}$ disc was set on the shaft midspan. An unbalance weight of $1 \mathrm{~g}$ was mounted. The tests were carried out at a rotational speed of $1000 \mathrm{rpm}$ (well below the shaft $2700 \mathrm{rpm}$ first critical speed). Various phase angles between the centrifugal force and the imposed displacement of the bearing mobile housing $\left(0^{\circ}, 45^{\circ}\right.$, $90^{\circ}, 135^{\circ}, 180^{\circ}, 225^{\circ}, 270^{\circ}, 315^{\circ}$ ) were considered. In these initial tests, the mobile housing was forced on a circular trajectory with a radius of $25 \mu \mathrm{m} .25$ scans per revolution for 10 revolutions were performed, corresponding to a sampling rate of $416.67 \mathrm{~Hz}$. Average temperature and lubricant pressure were respectively $22^{\circ} \mathrm{C}$ and $19.6 \mathrm{kPa}$.

\section{RESULTS}

The more interesting results are those with $0^{\circ}$ and $180^{\circ}$ phase angle between centrifugal exciting force and displacement imposed to the bearing housing. In Fig.9 the orbits covered by the shaft axis near the disc, with no control and with active feedforward control respectively, are shown.

The results indicate that with a phase angle of $180^{\circ}$ a reasonable reduction of orbit can be obtained. The orbit, even before the activation of the mobile bearing, isn't purely elliptical but rather curly. Among the causes of this peculiar orbit shape there is probably a super-synchronous force component which is more effective when the synchronous one is reduced.
In fact looking at the power spectrum of the displacement signal (Fig.10) peaks at $2 \mathrm{x}, 3 \mathrm{x}$ and $4 \mathrm{x}$ the fundamental frequency can be noticed. These harmonics may arise from the non-perfect uniformity of the shaft rotational speed forced by the motor, from misalignment, and from the different reaction of the piezoactuator/spring assembly in the two displacement directions.

The orbits are much greater than expected, even in the uncontrolled operating conditions. That is probably due to static unbalance related to a certain bowing of the shaft.

In Fig. 11 the result of the test performed imposing the optimal orbit of the housing calculated by simulation is shown. The orbit is flattened in one direction but it is rather large in the other direction. The result indicates that the data used in the simulation are far from the real ones and experimental identification of the bearing parameters is needed for control to be effective. In fact the calculated stiffness and damping coefficients obtained by applying the classical lubrication theory are not reliable because of the simplifying assumptions made on the lubricating film extension and on the linear dynamic behavior. In particular the effects of the supply holes and of the journal whirling motion on the lubricating film are neglected.

Initial experiences have also shown that the flow of lubricant trough the bearing was insufficient. During operation, the lubrication regime could be not hydrodynamic but mixed. Consequently some rig modifications have been made to reduce the pressure losses in the supply circuit. 

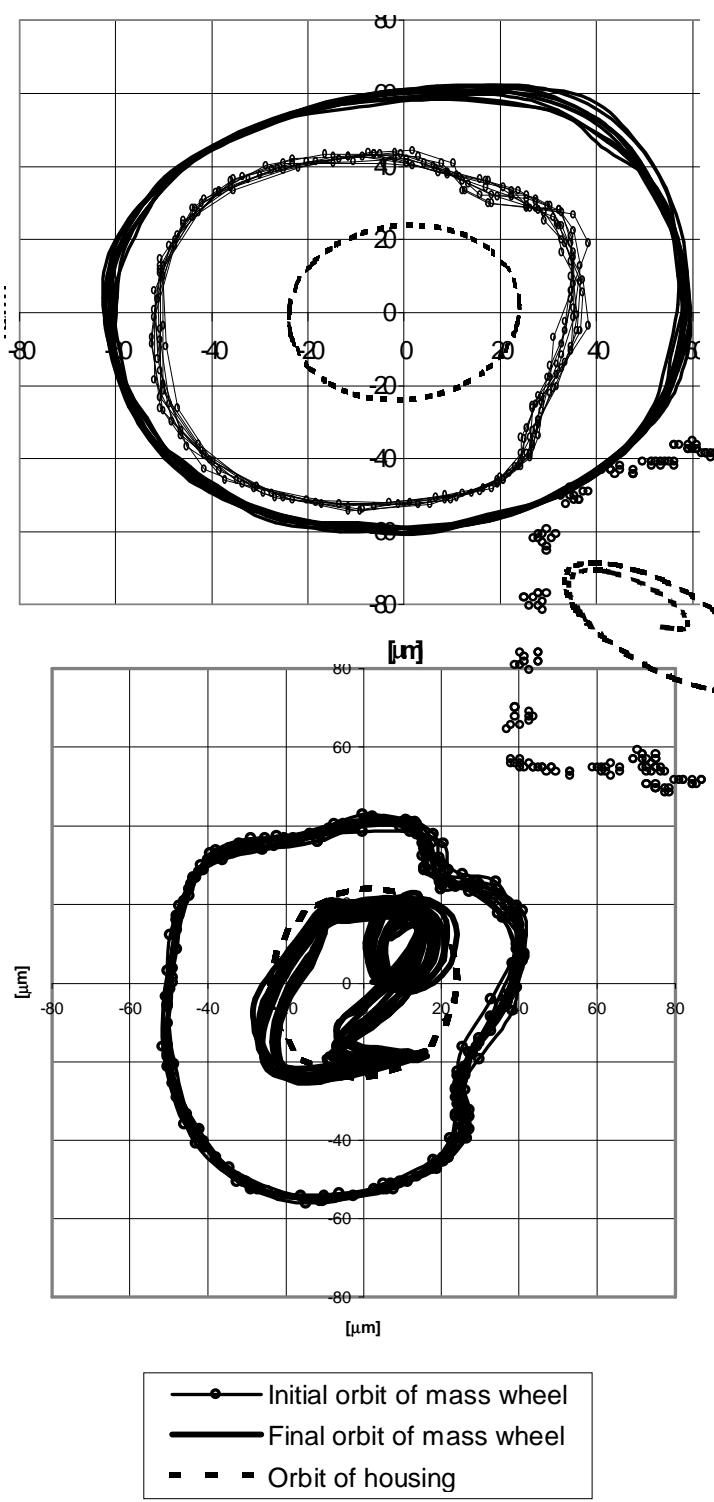

Fig. 9. Uncontrolled and controlled orbits: $0^{\circ}$ and $180^{\circ}$ phase angle

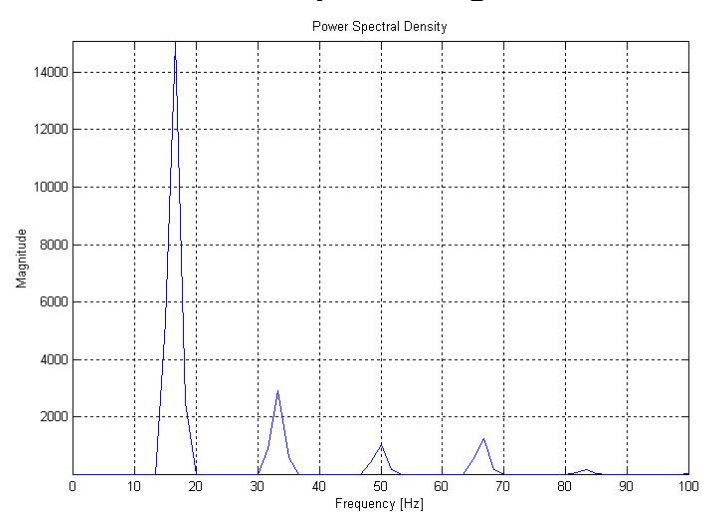

Fig.10. Power spectrum of a displacement signal $\left(180^{\circ}\right.$ phase angle)

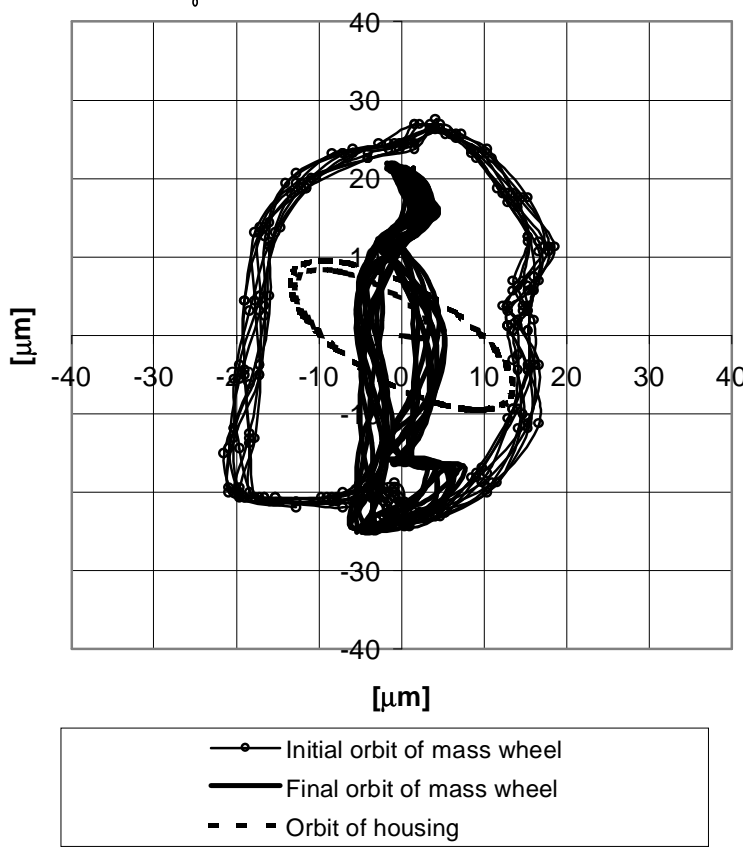

\section{Fig. 11. Initial orbit and orbits obtained moving the housing along the theoretical optimal orbit}

Particular attention must be paid to the number of scans used to represent the imposed displacement function. An insufficient number of scans may cause a jerking action of the piezoelectric actuators and consequently the generation of disturbing noise.

\section{SIMULATED IDENTIFICATION}

Experimental identification of the device is planned for the near future. At present a numerical simulation of the identification procedure has been carried out using a modulating function approach [7]. This technique has the advantage of being rather insensitive to noise. The idea is to suitably integrate over a time interval the measured signals and their derivatives multiplied by particular functions.

In order to identify the mobile hydrodynamic bearing the following equations of motions have to be considered:

$$
\left.\left.\left[\begin{array}{ll}
c_{x x} & c_{x y} \\
c_{y x} & c_{y y}
\end{array}\right]\left\{\begin{array}{l}
\dot{x}_{2}-\dot{x}_{3} \\
\dot{y}_{2}-\dot{y}_{3}
\end{array}\right\}+\left[\begin{array}{ll}
k_{x x} & k_{x y} \\
k_{y x} & k_{y y}
\end{array}\right]\left\{\begin{array}{l}
x_{2}-x_{3} \\
y_{2}-y_{3}
\end{array}\right\}=-\left[\begin{array}{cc}
k_{21} & 0 \\
0 & k_{21}
\end{array}\right]\left\{\begin{array}{l}
x_{1} \\
y_{1}
\end{array}\right\}-\left[\begin{array}{cc}
k_{22} & 0 \\
0 & k_{22}
\end{array}\right]\right\} \begin{array}{l}
x_{2} \\
y_{2}
\end{array}\right\}
$$

where the unknown variables are the bearing coefficients (4 for each equation). White noise is imposed to $x_{3}$ and $y_{3}$ (input of the system to be identified) and displacements $x_{1}, y_{1}$, $x_{2}$ and $y_{2}$ are measured. The members of the above equations are multiplied by the modulating functions and integrated over time to reduce noise. The number of equations can be increased in order to improve the averaged evaluation, 
considering different time intervals in a sufficiently long acquisition time.

The identification procedure was simulated numerically and the error between the displacements of the reference model to be identified and those of the model with the identified coefficients subjected to the same input was smaller than 8 microns. Thus the results are very encouraging.

\section{CONCLUSIONS AND FUTURE DEVELOPMENTS}

Preliminary tests have shown the possibility of controlling actively the vibrations of a slender shaft by suitably moving the housing of a plain hydrodynamic additional journal bearing. In fact the tests showed a reduction of the orbit of the disc mounted on the controlled shaft mid-span.

At present the field of implementation of these devices is limited to balancing of small size rotors, because of the characteristics of available piezoelectric actuators, in particular their limited stiffness and maximum displacement, but hopefully these limits will be overcome in the future.

The planned future developments of the research activity are the experimental identification of the active bearing, the elaboration of a feed-forward control algorithm.

\section{REFERENCES}

[1] Muszynska, A., Franklin, W.D., Bently, D.E., 1988, "Rotor Active "Anti-Swirl" Control", ASME Journal of Vibration, Acoustics, Stress, and Reliability in Design, 110, pp. 143-150.

[2] Ulbrich, H., 1992, "Active bearing support for rotating machine elements", Machine Vibration, 1, pp. 2-12.

[3] Santos, I.F., 1994, "Design and evaluation of two types of active tilting pad journal bearings", Proceedings of IUTAM Symposium: The Active Control of Vibration, University of Bath (UK), pp. 45-52.

[4] Krodkiewski, J. M., Sun, L., 1995, "Stability control of rotor-bearing system by an active journal bearing", Proc. Vibration and Noise, Venezia, pp. 217-225.

[5] Palazzolo, A. B., Jagannathan, S., Kascak, A. F., Montague, G. T., Kiraly, L. J., 1993, "Hybrid active vibration control of rotorbearing system using piezoelectric actuators", Journal of Vibration and Acoustics, Trans. of ASME, 115, pp. 111-119.

[6] Barret, T. S., Palazzolo, A. B., Kascak, A. F., 1993, "Active vibration control of rotating machinery using piezoelectric actuators incorporating flexible casing effects", International Gas Turbine and Aeroengine Congress and Exposition, Transactions of the ASME, Cincinnati, Ohio.

[7] Balestrino, A., Carmignani, C., Forte, P., Landi, A., 1996, "Rotor Balancing by means of active hydrodynamic bearings", Proc. XXV AIAS National Conference International Conference on Material Engineering, Gallipoli (LE), 4-7 Sept.1996, pp. 615-622.

[8] Bonneau, O., Lecoutre, E., Frêne, J., 1998, "Dynamic behavior of a rigid shaft mounted in an active bearing", Proceedings of ISROMAC-7 International Symposium on Transport Phenomena and Dynamics of Rotating Machinery, Honolulu, Hawaii (USA), pp. 30-37.

[9] Larsonneur, R., Herzog, R.J.P., 1994, "Feedforward compensation of unbalance: new results and application experiences", Proceedings of IUTAM Symposium: The Active Control of Vibration, University of Bath (UK), pp. 45-52.

[10]Fuller, C. R., Elliott, S. J., Nelson, P. A., “Active Control of Vibration", Academic Press Limited, London, 1996.

[11] Krämer, E., 1993, "Dynamics of rotors and foundation", Springer-Verlag, Berlin Heidelberg.

[12] Frêne, J., Nicolas, D., Deguerce, B., Berthe, D., Godet, M., 1990, "Hydrodynamic Lubrication, bearing and thrust bearing”, Elsevier, Tribology Handbook 33. 